\section{Chlamydia trachomatis infection: a challenge for the urologist}

\author{
Tommaso Cai, ${ }^{1}$ Sandra Mazzoli, ${ }^{2}$ \\ Nicola Mondaini, ${ }^{3}$ Gianni Malossini, ${ }^{1}$ \\ Riccardo Bartoletti ${ }^{3}$ \\ 1Department of Urology, Santa Chiara \\ Hospital, Trento; ${ }^{2}$ Sexually Transmitted \\ Disease Centre, Santa Maria Annunziata \\ Hospital; ${ }^{3}$ Department of Urology, \\ University of Florence, Italy
}

\begin{abstract}
The role of Chlamydia trachomatis $(\mathrm{Ct})$ in everyday clinical practice is now on the increase because $\mathrm{Ct}$ infections are the most prevalent sexually transmitted bacterial infections worldwide. Ct can cause urethritis, cervicitis, pharyngitis, or epididymitis, although asymptomatic infections are quite common. $\mathrm{Ct}$ infection remains asymptomatic in approximately $50 \%$ of infected men and $70 \%$ of infected women, with risk for reproductive tract sequelae both in women and men. A proper early diagnosis and treatment is essential in order to prevent persistent consequences. An accurate comprehension of the pathology, diagnosis and treatment of this entity is essential for the urologist. We review the literature about the new findings in diagnosis and treatment of $\mathrm{Ct}$ infection in sexually active young men.
\end{abstract}

\section{Introduction}

Sexually transmitted diseases (STDs) are among the first ten causes of unpleasant diseases in young adult males in developing countries and the second major cause of unpleasant diseases in young adult women, with an enormous health and economic consequences. ${ }^{1}$ Among these, Chlamydia trachomatis (Ct) is the most common sexually transmitted bacterium worldwide ${ }^{2}$ with over three million new infections per year. ${ }^{3}$ In particular, Chlamydia is the most frequently reported sexually transmitted infection in Europe and the number of cases is steadily increasing, with more than 255,000 cases in people below 25 years of age. ${ }^{4} \mathrm{Ct}$ infection could remain asymptomatic in about $70 \%$ of cases. $^{5-6} \mathrm{Ct}$ infection long-term effects include ectopic pregnancy and tubal inflammation with subsequent infertility. ${ }^{6-7}$ Absence of symptoms increases the risk of infecting sexual partners and may cause long-term complications in men too, such as poor quality of semen and infertili- ty. ${ }^{5,7}$ Several factors contribute to make difficult detecting $\mathrm{Ct}$ by a conventional analysis. ${ }^{8}$ To date, the DNA recombination techniques are universally accepted as the gold standard to evaluate the presence of $\mathrm{Ct}$ in biologic samples. ${ }^{9}$ However, immunologic markers of $\mathrm{Ct}$ infection such as immunoglobulin A (IgA) antibody and cytokines have been detected in total ejaculate and seminal plasma samples to demonstrate their role in monitoring men with $\mathrm{CP}{ }^{10-11}$ Moreover, the proper treatment of urological $\mathrm{Ct}$ infection is not totally indicated. For these reasons, Ct represents a challenge for the urologist both for diagnostic and treatment. We summarize the most current developments in the diagnostics and therapeutic approaches in Ct infections in sexually active young men. ${ }^{12}$

\section{Methods of searching}

We conducted a search of the English-language literature from 1960 through December 2010 with use of the Medline computerized database of the US National Library of Medicine (http//www.ncbi.nlm.nih.gov/pubmed). The Medline search has been divided into two sections: diagnosis and therapy. The first review section about diagnosis has been carried-out by using the following Medical Subject Headings and free text terms: Chlamydia trachomatis, and chlamydia infections (exploded) were combined with the terms diagnosis, urine, urethral swa, total ejaculate, serum, antibodies, prostate massage and then limited to humans, male and young adult: 19-24 years. The second review section about therapy has been carried-out by using the following Medical Subject Headings and free text terms: Chlamydia trachomatis, and chlamydia infections (exploded) were combined with the terms treatment, therapy, antibiotic, drug, quinolones, tetracycline and then limited to humans, male and young adult: 19-24 years. Moreover, we searched reference lists of articles to identify potential additional references. All original paper and review studies of $\mathrm{Ct}$ diagnosis and treatment in young adult have been considered for this review. We considered also guidelines from the National Institute for Health and Clinical Excellence and the European Centre for Disease Prevention and Control, the US Centers for Disease Control and Prevention, and World Health Organization.

\section{Results}

\section{Chlamydia trachomatis diagnosis}

From an initial literature search with 188 unique citations, a total of 27 articles were selected for the present review. A matched research between Chlamydia trachomatis, and chlamydia infections (exploded) and the fol-
Correspondence: Tommaso Cai, Department of Urology, Santa Chiara Hospital, Largo Medaglie d'Oro 9, Trento, Italy.

Tel. +39.0461 .903306 - Mobile +39.3339864943

Fax: +39.0461 .903101$

E-mail: ktommy@libero.it

Key words: chlamydia trachomatis, prostatitis, sexually transmitted diseases, infection, quinolones, sexually active young men.

Received for publication: 16 June 2011. Accepted for publication: 27 August 2011.

This work is licensed under a Creative Commons Attribution NonCommercial 3.0 License (CC BYNC 3.0).

(C) Copyright T. Cai et al., 2011

Licensee PAGEPress, Italy

Microbiology Research 2011; 2:e14

doi:10.4081/mr.2011.e14

lowing terms total ejaculated and prostate mas sage has not found any items.

Diagnosis of $\mathrm{Ct}$ infection can be made by using:
- Direct detection
- Indirect detection

\section{Direct detection}

Ct is an obligate intracellular bacterium and cell culture remains a reference method (about $100 \%$ specificity). ${ }^{13}$ However, all Authors are agree that it is not recommended for routine use, due to its lack of sensitivity, its technical complexity and the long turn-around time. ${ }^{14}$ Other Authors suggest that $\mathrm{Ct}$ can be found by using antigen-based detection methods. ${ }^{13}$ In particular, direct fluorescent staining with monoclonal antibodies (DFA) and enzyme immunoassay (EIA). EIA tests are more reproducible than DFA, and the sensitivity of the best EIA is comparable to that of culture but lower than that of nucleic acid amplification tests (NAATs), due to the cross-reactions with the lipopolysaccharide (LPS) of other microorganisms. ${ }^{13}$ Recently, Mahilum-Tapay and co-workers, evaluated the performance of the Chlamydia Rapid Test, a new assay developed at the Diagnostics Development Unit, University of Cambridge. ${ }^{15}$ They compared sensitivity, specificity, positive predictive value, and negative predictive value of the Chlamydia Rapid Test with the gold standard test for Ct infections (NAATs). ${ }^{15}$ In this study, they found a good diagnostic performance: sensitivity $83.5 \%$, specificity $98.9 \%$, positive predictive value $86.7 \%$, and negative predictive value $98.6 \%{ }^{15}$ However, NAATs are the tests of choice for the diagnosis of $\mathrm{Ct}$ genital infections. ${ }^{13}$ In everyday clinical practice, several commercial NAATs are available, and make use of different technologies: PCR and real-time PCR (Roche Diagnostics, 
Abbott, IL, USA); strand displacement amplification (Becton Dickinson, NJ, USA); transcription-mediated amplification (Gen Probe); and nucleic acid sequence-based amplification (bioMerieux, Nancy L'Etoile, France). ${ }^{13,16}$ These assays are automated and can be used for screening programmes and for the detection of $\mathrm{Ct}$ and Neisseria gonorrhoeae in the same specimen. ${ }^{13}$ We currently used Roche COBAS AMPLICOR CT/NG reagents kits and instruments (Roche Molecular Systems,Branchburg, NJ, USA) with good level of accuracy. ${ }^{8}$ NAATs tests generally show two important drawbacks: the cost and the presence of inhibitors in specimens. However, they show a high specificity. ${ }^{13}$ Finally, in 2006, a new C. trachomatis variant belonging to serovar $\mathrm{E}$, with a 377 -bp deletion in the cryptic plasmid, was described in Sweden. ${ }^{17}$ This new variant can obviously not be detected by amplification tests targeting the deleted area, but can be detected by amplification targeting a chromosomal gene, e.g. ompA or a rRNA gene. ${ }^{17}$ However, new versions of the COBAS Taqman v2.0 test and of the Abbott test allow simultaneous detection of the cryptic plasmid and of ompA, and simultaneous detection of two different regions of the cryptic plasmid, respectively. ${ }^{17}$

\section{Indirect detection}

A recent review by Persson suggested that serology is useful only in some cases of $\mathrm{Ct}$ infection and in seroepidemiological studies. ${ }^{18}$ On the other hand, recent evidences showed that anti-Ct immunoglobulin A (IgA) in association with interleukin 8 (IL-8) evaluation appear to be the best immunologic markers of chronic chlamydial prostatitis status. ${ }^{8}$ Mazzoli and co-workers, highlighted, in 78 consecutive patients with a diagnosis of chronic prostatitis due to $\mathrm{Ct}$ infection by IPAzyme Chlamydia IgG/IgA by Savyon Diagnostics (Ashdod, Israel), an immune-peroxidase test, the role of immune system activation in the pathophysiology of chronic prostatitis due to $\mathrm{Ct}$ infection and that seminal IL-8 and mucosal IgA levels specific to $\mathrm{Ct}$ antigens appear to be the best immunologic markers of chronic chlamydial prostatitis status. ${ }^{8}$ They, however, did not showed any role of serum anti-Ct immunoglobulin in Ct infection diagnosis. ${ }^{8}$

\section{Chlamydia trachomatis therapy}

From an initial literature search with 164 unique citations, a total of 18 articles were selected for the present review. A matched research between Chlamydia trachomatis and chlamydia infections (exploded) and the following terms quinolones has not found any items.

A recent review by suggested that antimicrobial groups effective against $\mathrm{Ct}$ include the macrolides, tetracyclines, quinolones and penicillins. ${ }^{19}$ The European Urological Association and the Centers for Disease Control and Prevention guidelines suggested that doxycycline and azithromycin are considered to be equally effective in the treatment of chlamydial infections. ${ }^{2,12}$ However, in management and treatment of patients affected by $\mathrm{Ct}$ infections the following factors should be taken into account: i) Chlamydia are only metabolically active in the host cell and therefore only targeted intracellularly by antibiotics; ii) Intracellularly accumulated antibiotics are tetracyclines, macrolides and quinolones. ${ }^{2,12}$ Even if doxycycline and azithromycin are the most widely prescribed drugs in Ct infections treatment and recommended as the primary approach, other fluoroquinolones such as ofloxacin or levofloxacin are suggested as alternative drugs. ${ }^{2}$ Moreover, although little is known about $\mathrm{Ct}$ survival in the presence of fluoroquinolones, it is well known that after multiple cultivation passages resistant mutant for some fluoroquinolones were determined.$^{20}$ In a recent report, Smelov and co-workers suggested that ofloxacin could be recommended as the primary drug in the treatment of chlamydia-infected patients with $\mathrm{CP}$, due to its pharmacokinetic parameters. ${ }^{20}$ Moreover, the same Authors stated that the decision on the prescription of pefloxacin or lomefloxacin should be made individually, but ciprofloxacin treatment is not suggested. ${ }^{20}$

The authors, however, concluded that the conditions of in vitro susceptibility studies are incompatible with the infection as it occurs in vivo even if could be useful to include investigations for antibiotic susceptibility in every patient prior to treatment. ${ }^{20}$ In a recent study Cai et al., by means of a prospective, randomized and open-label study on 221 patients affected by chronic prostatitis due to Ct infection who had undergone oral administered prulifloxacin $600 \mathrm{mg}$ once daily for 14 days or doxycycline $100 \mathrm{mg}$ orally twice daily for 21 days, found that prulifloxacin was equivalent to the standard therapy. ${ }^{21}$ In this study, moreover, the Authors showed that prulifloxacin was superior over standard therapy in microbiological efficacy rates in terms of mucosal IgA and IL-8 levels decreasing. ${ }^{21}$ This effect should be probably, due to an anti-inflammatory effect of quinolones.

The role of pro-inflammatory cytokines such as IL-6 or IL-8 in Ct infection is well established, discussed and used not only in the diagnosis phase but also in management and ther-

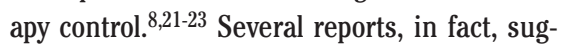
gested that IL-8 evaluation should be used, not only as a Ct infection marker, but also as a marker of therapy efficacy. ${ }^{8,21}$ The role of molecular markers in the management of $\mathrm{Ct}$ infections is, thus, clinically useful and suggested. Mazzoli et al., recently, demonstrated that patients who had reported the higher mean value for IL- 8 and massive presence of mucosal IgA, making evident a strong inflammation and a correlation with the higher level of pain and a worse quality of life, with a significant correlation between IL-8 and IgA values and NIH-CPSI subscale scores. ${ }^{8}$ Moreover, in the clinical practice, Cai et al. found a good relationship between IL-8 and NIH-CPSI, demonstrating that an improvement in QoL (NIH-CPSI decreasing) is related to a decrease in IL-8 levels after theapy. ${ }^{21}$ In addition, an important role should be given to mucosal IgA anti-Ct evaluation. Some authors have demonstrated, in animal model, that a high production of IgA in genital tract secretions seems related to the presence, persistence, and accumulation of Th2 MoPn cells in the genital tract during chronic infections, with the consequent inability to clear the infection. ${ }^{23}$ In particular, the presence of an active chronic infection in patients affected by Ct infection is also well correlated to the presence of high levels of anti-Heat Shock Protein 60 (anti-HSP60) mucosal IgA antibodies, as proved by the high percentage of IgA positive patients showing in western blot analysis an immunoreactions towards high molecular weight proteins, especially MOMP2 and HS60. ${ }^{8}$ This anti-HSP60 immunization suggests chronic or repeated stimulation from an endemic source of the microrganism, ${ }^{24}$ proved by the presence of CT DNA found in young sexually active patients affected by chronic prostatitis due to $\mathrm{Ct}$ infection. Finally, any correlation between serum IgA or IgG and the other Ct maker of infections, or any significant change before or after therapy, have been reported. ${ }^{21}$ In fact, the role of serum IgA or IgG anti-Ct in early diagnosis of infection and early treatment has been reported for women's infection, but not, up to the moment, for males. ${ }^{25}$ Clinical trials carried out with the aim to test the microbiological or clinical efficacy of antibiotics different to quinolones, are very few. In a recent experience, Takahashi and coworkers, showed a good clinical cure rate $(77 \%)$ by using a single-dose of azithromycin (1000 mg) for 13 patients with urethritis due to $\mathrm{Ct}$ infection. ${ }^{26}$ The Authors concluded that a single-dose azithromycin regimen was well tolerated and eradicated potential pathogens of NGU26. This study, however, has been carried out in a small cohort of patients and in inhomogeneous group of patients. In conclusions, the potential of $\mathrm{Ct}$ to develop antimicrobial resistance has not been well studied, although some case reports suggest resistance as a cause of treatment failure. ${ }^{27-29}$ The Table 1 summarized all randomized, controlled studies in management of $\mathrm{Ct}$ infection in young male patients. 
Table 1. Randomized, controlled, clinical trials.

\begin{tabular}{|c|c|c|c|c|c|}
\hline Author/year & Drugs & $\begin{array}{l}\text { Patient } \\
\text { number }\end{array}$ & $\begin{array}{l}\text { Clinical } \\
\text { efficacy } \\
(\%)\end{array}$ & $\begin{array}{c}\text { Microbiological } \\
\text { efficacy } \\
(\%)\end{array}$ & $\begin{array}{l}\text { Microbiological } \\
\text { markers }\end{array}$ \\
\hline Cai T. (2009) & Prulifloxacin/Doxicicline & 221 & 82.5 & 47.7 & IL-8/IgA \\
\hline Whatley J.D. (1991) & Azithromycin/Doxicicline & 62 & - & - & - \\
\hline Jeskanen L. (1989) & Ciprofloxacin/Doxicicline & 200 & 74 & - & - \\
\hline Stolz E. (1986) & Ciprofloxacin 250/Ciprofloxacin 500 & 212 & 100 & 100 & - \\
\hline
\end{tabular}

\section{Conclusions}

In conclusion, additional studies of the effectiveness of the common diagnostic tests for $\mathrm{Ct}$ infection would be valuable. In addition, better natural history data on the timing of male genital Ct infection and development of more accurate, noninvasive tools to assess chlamydial sequelae are essential to plan a correct treatment schedule. Finally, clinical trials should be planned in order to evaluate the real frequency of $\mathrm{Ct}$ resistance to standard therapy.

\section{References}

1. Da Ros CT, Schmitt Cda S. Global epidemiology of sexually transmitted diseases. Asian J Androl 2008;10:110-4.

2. Centers for Disease Control and Prevention. STD Treatment Guidelines, 2002. Available from: http://www.cdc.gov/ std/treatment/default-2002.htm

3. Groseclose SL, Zaidi AA, DeLisle SJ, et al. Estimated incidence and prevalence of genital Chlamydia trachomatis infections in the United States, 1996. Sex Transm Dis 1999;26:339-44.

4. European Centre for Disease Prevention and Control. Most common STI in Europe. Available from: http:/www.ecdc.europa.eu/ en/healthtopics/spotlight/chlamydia/Pages /KeyMessagel.aspx

5. Mazzoli S, Cai T, Addonisio P, et al. Chlamydia trachomatis Infection Is Related to Poor Semen Quality in Young Prostatitis Patients. Eur Urol 2009;57:70814.

6. Park IU, Amey A, Creegan L, et al. Retesting for Repeat Chlamydial Infection: Family Planning Provider Knowledge, Attitudes, and Practices. J Womens Health (Larchmt) 2010;19:1139-44.

7. Schachter J, Caldwell HD. Chlamydiae. Annu Rev Microbiol 1980;34:285-309.

8. Mazzoli S, Cai T, Rupealta V, et al. Interleukin 8 and anti-chlamydia trachomatis mucosal IgA as urogenital immunologic markers in patients with $\mathrm{C}$. Trachomatis prostatic infection. Eur Urol 2007;51:1385-93.

9. Petzold D, Gross G, editors. Diagnostik und Therapie sexuell übertragbarer Krankheiten. Leitlinien 2001 der Deutschen STD-Gesellschaft. Berlin; Springer; 2001.

10. Wagenlehner FME, Weidner W, Naber KG. Chlamydial infections in urology. World $\mathrm{J}$ Urol 2006;24:4-12.

11. Ostaszewska-Puchalska I, ZdrodowskaStefanow B, Badyda J, et al. Antichlamydial antibodies in the serum and expressed prostatic secretion in prostatitis. Arch Immunol Ther Exp (Warsz) 2004;52:277-83.

12. Naber KG, Bergman B, Bishop MC, et al. EAU guidelines for the management of urinary and male genital tract infections. Urinary Tract Infection (UTI) Working Group of the Health Care Office (HCO) of the European Association of Urology (EAU). Eur Urol 2001;40:576-88.

13. Bébéar C, de Barbeyrac B. Genital Chlamydia trachomatis infections. Clin Microbiol Infect 2009;15:4-10.

14. Black CM. Current methods of laboratory diagnosis of Chlamydia trachomatis infections. Clin Microbiol Rev 1997;10:160-84.

15. Mahilum-Tapay L, Laitila V, Wawrzyniak $\mathrm{JJ}$, et al. New point of care Chlamydia Rapid Test--bridging the gap between diagnosis and treatment: performance evaluation study. BMJ. 2007;335:1190-4.

16. Leber AL, Hall GS, LeBar WD. Nucleic acid amplification tests for detection of Chlamydia trachomatis and Neisseria gonorrhoeae. In: Sharp SE, editor. Cumitech 44. Washington, DC: ASM Press; 2006. pp. 1-38.

17. Ripa T, Nilsson P. A variant of Chlamydia trachomatis with deletion in cryptic plasmid: implications for use of PCR diagnostic tests. Euro Surveill 2006;11:E061109.

18. Persson K. The role of serology, antibiotic susceptibility testing and serovar determination in genital chlamydial infections. Best Pract Res Clin Obstet Gynaecol 2002;16:801-14.

19. Manavi K. A review on infection with Chlamydia trachomatis. Best Pract Res Clin Obstet Gynaecol 2006;20:941-51.
20. Smelov V, Perekalina T, Gorelov A, et al. In vitro activity of fluoroquinolones, azithromycin and doxycycline against chlamydia trachomatis cultured from men with chronic lower urinary tract symptoms. Eur Urol 2004;46:647-50.

21. Cai T, Mazzoli S, Addonisio P, et al. Clinical and microbiological efficacy of prulifloxacin for the treatment of chronic bacterial prostatitis due to Chlamydia trachomatis infection: results from a prospective, randomized and open-label study. Methods Find Exp Clin Pharmacol 2010;32:39-45.

22. Kokab A, Akhondi MM, Sadeghi MR, et al. Raised inflammatory markers in semen from men with asymptomatic chlamydial infection. J Androl 2010;31:114-20.

23. Martínez-Prado E, Camejo Bermúdez MI. Expression of IL-6, IL-8, TNF-alpha, IL-10, HSP-60, anti-HSP-60 antibodies, and antisperm antibodies, in semen of men with leukocytes and/or bacteria. Am J Reprod Immunol 2010;63:233-43.

24. Sziller I, Witkin SS, Ziegert M, et al. Serological responses of patients with ectopic pregnancy to epitopes of the Chlamydia trachomatis $60 \mathrm{kDa}$ heat shock protein. Hum Reprod 1998;13:1088-93.

25. Mascellino MT, Ciardi MR, Oliva A, et al. Chlamydia trachomatis detection in a population of asymptomatic and symptomatic women: correlation with the presence of serological markers for this infection. New Microbiol 2008;31:249-56.

26. Takahashi S, Matsukawa M, Kurimura Y, et al. Clinical efficacy of azithromycin for male nongonococcal urethritis. J Infect Chemother 2008;14:409-12.

27. Somani J, Bhullar VB, Workowski KA, et al. Multiple drug-resistant Chlamydia trachomatis associated with clinical treatment failure. J Infect Dis 2000;181:1421-7.

28. Mourad A, Sweet RL, Sugg N, Schachter J. Relative resistance to erythromycin in Chlamydia trachomatis. Antimicrob Agents Chemother 1980;18:696-8.

29. Lefevre JC, Lepargneur JP. Comparative in vitro susceptibility of a tetracycline-resistant Chlamydia trachomatis strain isolated in Toulouse (France). Sex Transm Dis 1998;25:350-2. 